\title{
Identification of Optimal Light Path in a WDM Network
}

\author{
Dil Afroza Jerin \\ Department of Information and Communication \\ Engineering \\ Pabna University of Science and Technology \\ Pabna, Bangladesh
}

\author{
Md. Omar Faruk \\ Department of Information and Communication \\ Engineering \\ Pabna University of Science and Technology \\ Pabna, Bangladesh
}

\begin{abstract}
A WDM network based on dynamic light-path allocation, we have to take into consideration the physical topology of the WDM network and the traffic requirements. The physical topology is defined by the nodes, typically computers that generate data to be transmitted where data is needed, the optical routers that determine how the optical signals are sent towards their respective destinations, and the fiber connections that provide the physical medium for communication. When we want to design an optical network, we think about optimal or best path for installing the network. According to the result of this research, the power budget and quality factor have been calculated from the given networks. The result shows that the path is the best one due to less power budget and high quality factor value and we shall also show the characteristics of the power budget and quality factor from the graph which is simulated in MATLAB.
\end{abstract}

Keywords-WDM network, Quality factor, Power budget, Receiver sensitivity, Transmit power

\section{INTRODUCTION}

An optical fiber is a flexible, transparent strand of very pure glass that acts as a light pipe to transmit light between two ends of the fiber. Optical fibers have a core surrounded by a cladding layer made of dielectric material. The optical signals in the core are confined by establishing a refractive index that is greater than the cladding. Optical fibers are used as a medium for telecommunication and networking. Light in a fiber optic cable travels through a core by constantly bouncing from the cladding, a principle termed total internal reflection. As the cladding does not absorb any light from the core, light waves travel longer distances. [1] Power budget refers to the amount of loss a data link can tolerate while maintaining proper operation. In power budget calculation different types of losses are also be calculated such as connector loss, splice loss, fiber loss. Attenuation causes reduction in signal strength or light power over the distance (length) of the light-carrying medium. Fiber attenuation is measured in decibels per kilometer $(\mathrm{dB} / \mathrm{km})$. Connector and splice loss in optical fiber is caused by different factors, which includes lateral and axial misalignment that occurs when the axes of the two fibers are offset in a perpendicular direction and angular misalignment which occurs when the axes of two connected fibers are no longer parallel. Coupling losses due to fiber alignment depend on fiber type, core diameter, and the distribution of optical power among propagating modes. Optical fiber offers better performance than other transmission media because it provides huge bandwidth with low attenuation. This allows signals to be transmitted over longer distances by using less regenerators or amplifiers, which reduces the cost and improves signal reliability. The recent developments in optical devices and network technologies use multiple optical signals on the same fiber is called wavelength division multiplexing (WDM). In WDM network design we face a problem to determine the optimal light-path from a set of all possible paths in a network topology. Based on the power budget calculations and quality factor calculations we can easily find the best light path. In this thesis, we do the same thing and simulated this result into MATLAB and get the different types of graphs of power budget and quality factor. We also find out the relation between power budget and quality factor. A connectivity matrix is always a square matrix. a number of rows and cells equivalent to the number of nodes in the network. Each cell representing a connection between two nodes receives a value of 1 . Each cell that does not represent a direct connection gets a value of $0 .[5]$

\section{METHODOLOGY}

Methodology is the systematic, theoretical analysis of the methods applied to a field of study. It comprises the theoretical analysis of the body of methods and principles associated with a branch of knowledge.

\section{A. Power Budget}

Power budget refers to the amount of loss a data link can tolerate while maintaining proper operation. In other words, it defines the amount of optical power available for successful transmitting signal over a distance of optical fiber. Power budget is the difference between the minimum (worst case) transmitter output power and the maximum (worst case) receiver input required. The calculations should always assume the worst-case values, in order to ensure the availability of adequate power for the link, which means the actual value will always be higher than this.[2] Optical power budget is measured by $\mathrm{dB}$, which can be calculated by subtracting the minimum receiver sensitivity from the minimum transmit power:

$$
\mathrm{P}_{\mathrm{B}}(\mathrm{dB})=\mathrm{P}_{\mathrm{TX}}(\mathrm{dBm})-\mathrm{P}_{\mathrm{RX}}(\mathrm{dBm})
$$

Power Budget Equation for Single link, using power budget equation (1), we can write

$$
\mathrm{Pb}(\mathrm{i}, \mathrm{j})=((((\mathrm{Pt}-\mathrm{Rs})-\alpha \mathrm{f} * \mathrm{~L})-\mathrm{Lc})-\mathrm{sm})
$$


Where, $\mathrm{Lc}=\alpha \mathrm{f} * \mathrm{~L}+\alpha \mathrm{c} * \mathrm{~N} 1+\alpha \mathrm{s} * \mathrm{~N} 2$

(3) [3]

A fiber link pair $(i, j)$ where, $i$ and $j$ represents source and destination nodes respectively. $P t$ is the power launch from source node $i$ into the fiber. $R s$ is receiver sensitivity. Optical communication system uses a bit error rate (BER) value to specify performance requirements. To achieve a desired BER a minimum average optical power value must arrive at the receiver end. This value is called receiver sensitivity.

$\alpha f$ is the fiber attenuation constant, $L$ is length of fiber in between source and destination.

$L c$ is link loss, $\alpha c$ is connector's loss, $\alpha$ s is splice's loss and $\mathrm{N} 1$ is the number of connectors and N2 is the number of splices used.

$\mathrm{Sm}$ is the system margin taken so that it will incorporate for component aging, temperature fluctuations and losses arising from components that might be added in future. Power Budget Equation for Multiple Links, The overall power budget (Pboverall) is given by

\section{Pboverall $=P t-R s-S m-\sum_{p \in k, l}^{n} L c(k, l)$}

Where, $p €$ all possible light-path and $(k, l)$ are node pair.[4]

\section{B. Quality Factor}

Q-Factor of a light-path is defined as the ratio of output power relative to input power. It is normalized by dividing the value of Q-Factor with maximum value of Q-factor possible. It is expressed in percentage. So $100 \%$ Q-Factor means light-path has the highest Q-Factor and the light-path corresponding to this value of Q-Factor will be the best lightpath. To maximize the Q-Factor we need to maximize the output power for constant value of input power. To maximize the Q-Factor we need to maximize the output power for constant value of input power. We know that output power received is the attenuated version of input power due to attenuation loss, splice loss and connector loss. So we should try to minimize the losses in the optical fiber communication. Losses can be reduced by selecting the best components like connectors, splices and optical fiber which are having minimum power loss values. Out of all possible light-paths, the light-path having minimum power loss should be selected as optimal light-path. Q-Factor is defined for a light-path as the ratio between output power and input power.[4]

If $P$ in is the input power, Pout is the output power and $P b$ is the overall power budget of a light-path having multiple links, then we can propose to define Q-Factor $(Q F)$ as

$$
Q F=\frac{\text { Pout }_{\text {Pin }}}{\text { Pin }}=(\text { pin }-p b) / \text { pin }
$$

\section{NETWORK DESIGN AND CALCULATION}

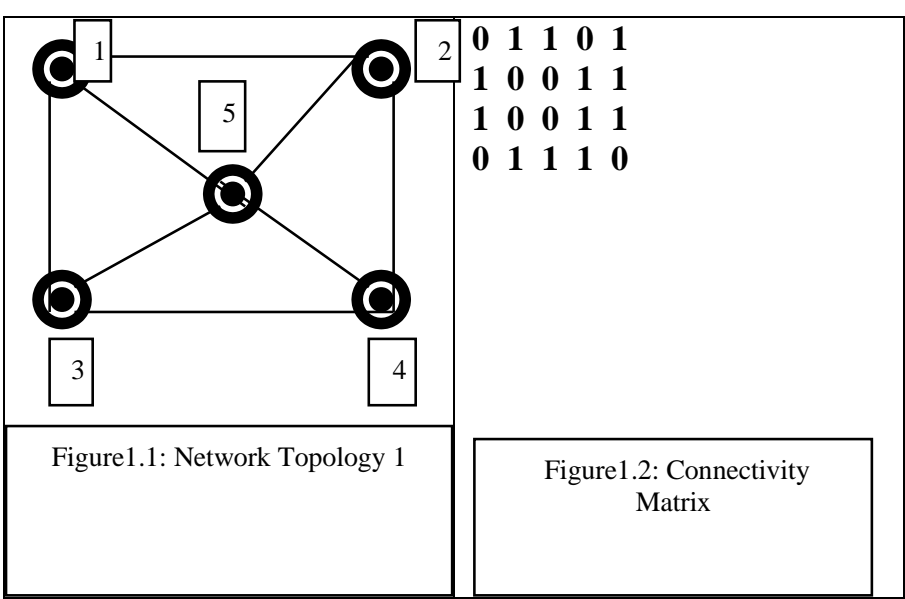

Table: 1.1: All possible path's power budget and quality factor values are

\begin{tabular}{|c|c|c|c|}
\hline $\begin{array}{l}\text { All Possible } \\
\text { Length ofPath }(\mathrm{km})\end{array}$ & Paths & $\begin{array}{c}\text { Power } \\
\text { Budget }(\mathrm{db})\end{array}$ & $\begin{array}{c}\text { Quality Factor } \\
(100 \%)\end{array}$ \\
\hline 1. $1-2-4$ & 30 & 50.5 & -152 \\
\hline $1-3-4$ & 130 & .5 & 97.5 \\
\hline $1-5-4$ & 90 & 20.8 & -4 \\
\hline $1-3-5-4$ & 125 & 2.70 & 86.5 \\
\hline 5. $1-3-5-2-4$ & 130 & -0.1 & * \\
\hline 6. $1-5-2-4$ & 95 & 17.1 & 14.5 \\
\hline
\end{tabular}

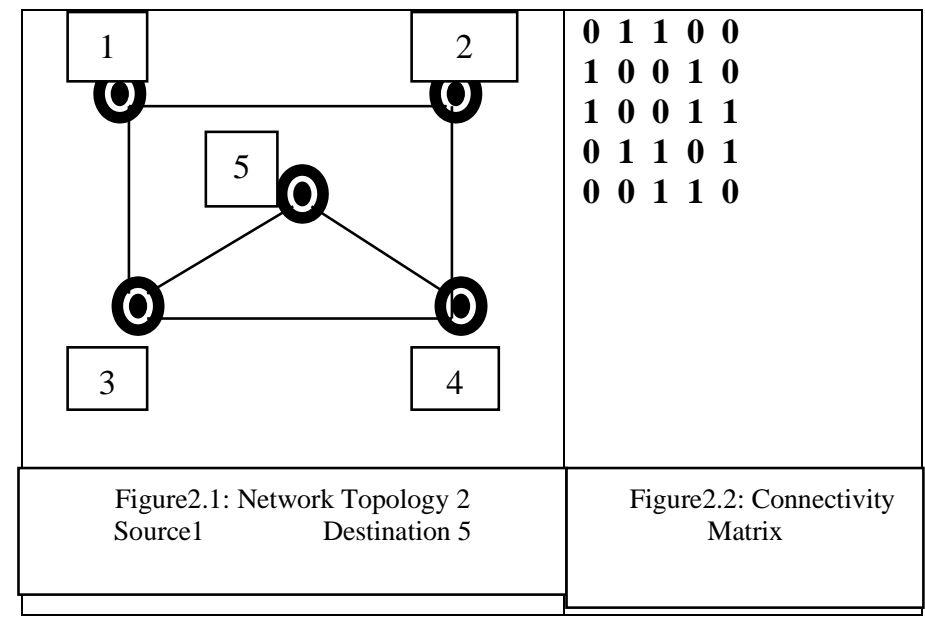

Table: 2.1: All possible path's power budget and quality factor values are given in a table

\begin{tabular}{|cl|c|c|c|}
\hline All Possible Paths & $\begin{array}{c}\text { Length of } \\
\text { paths(km) }\end{array}$ & $\begin{array}{c}\text { Power } \\
\text { Budget }(\mathrm{db})\end{array}$ & $\begin{array}{c}\text { Quality } \\
\text { Factor } \\
(100 \%)\end{array}$ \\
\hline 1. & $1-3-5$ & 78 & 6.5 & 50 \\
\hline 2. & $1-3-4-5$ & 83 & 3.4 & 74 \\
\hline 3. & $1-2-4-5$ & 87 & 11.4 & 12.3 \\
\hline 4. & $1-2-4-3-5$ & 122 & -16.7 & $*$ \\
\hline
\end{tabular}




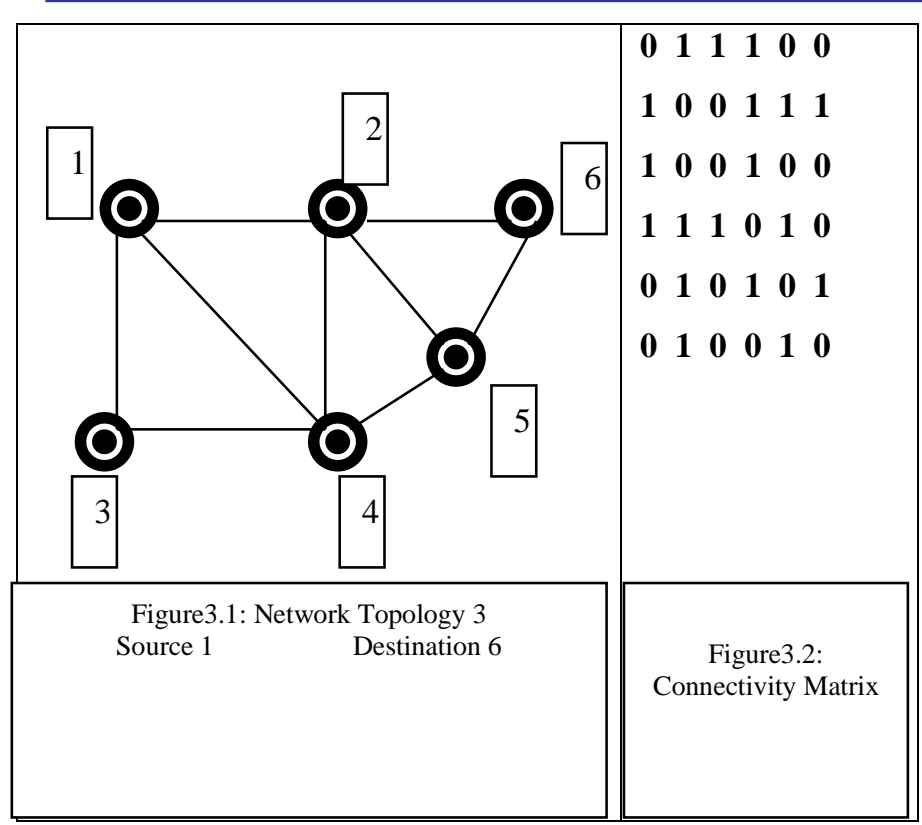

Table: 3.1: All possible path's power budget and quality factor values are given in a table.

\begin{tabular}{|c|c|c|c|}
\hline $\begin{array}{c}\text { All Possible } \\
\text { Paths }\end{array}$ & $\begin{array}{c}\text { Length of Paths } \\
(\mathrm{km})\end{array}$ & $\begin{array}{c}\text { Power Budget } \\
(\mathrm{db})\end{array}$ & $\begin{array}{c}\text { Quality Factor } \\
(100 \%)\end{array}$ \\
\hline $1-2-6$ & 1.3 & 57.98 & -44 \\
\hline $1-2-5-6$ & 70.8 & 29.58 & 26 \\
\hline $1-2-4-5-6$ & 66.4 & 30.74 & 23 \\
\hline $1-3-4-2-6$ & 205.5 & -24.9 & $*$ \\
\hline $1-4-5-6$ & 72.6 & 28.26 & 29 \\
\hline $1-4-2-6$ & 37.5 & 42.9 & -7 \\
\hline $1-4-2-5-6$ & 107 & 14.5 & 63 \\
\hline
\end{tabular}

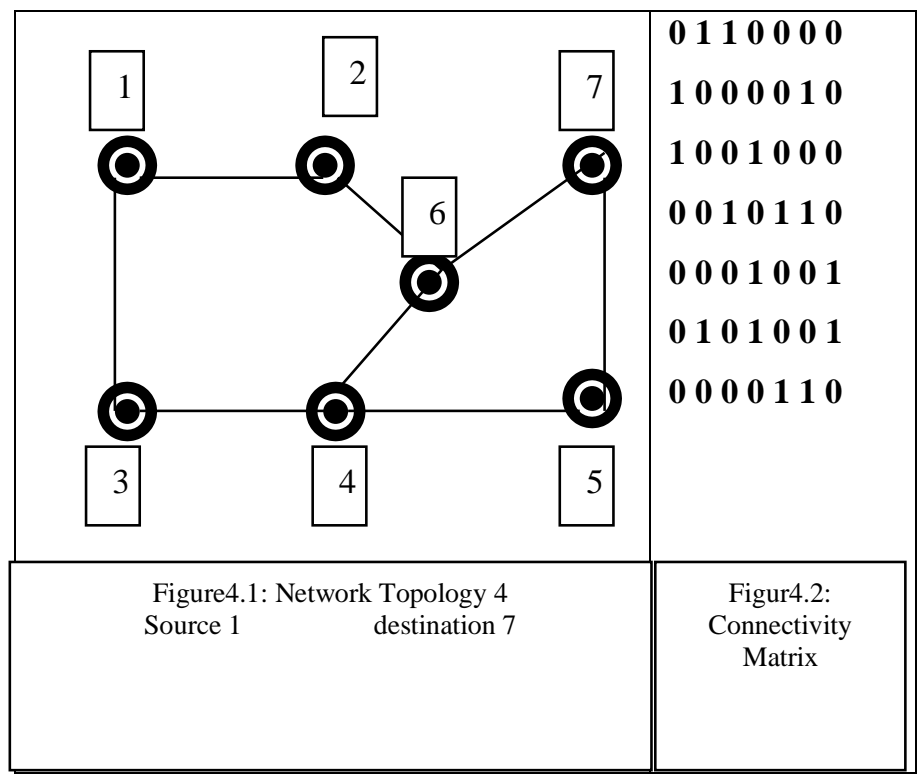

Table: 4.1: All possible path's power budget and quality factor values are given in a table.

\begin{tabular}{|cl|l|l|l|}
\hline All Possible Paths & $\begin{array}{l}\text { Length of } \\
\text { Paths }(\mathrm{km})\end{array}$ & $\begin{array}{l}\text { Power Budget } \\
(\mathrm{db})_{-}\end{array}$ & $\begin{array}{l}\text { Quality Factor } \\
(100 \%)\end{array}$ \\
\hline 1. & $1-2-6-7$ & 49 & 8.3 & 44 \\
\hline 2. & $1-3-4-6-7$ & 41 & 12.4 & 17 \\
\hline 3. & $1-3-4-5-7$ & 38 & 12.7 & 15 \\
\hline 4. & $1-2-6-4-5-7$ & 58 & 4.1 & 73 \\
\hline
\end{tabular}

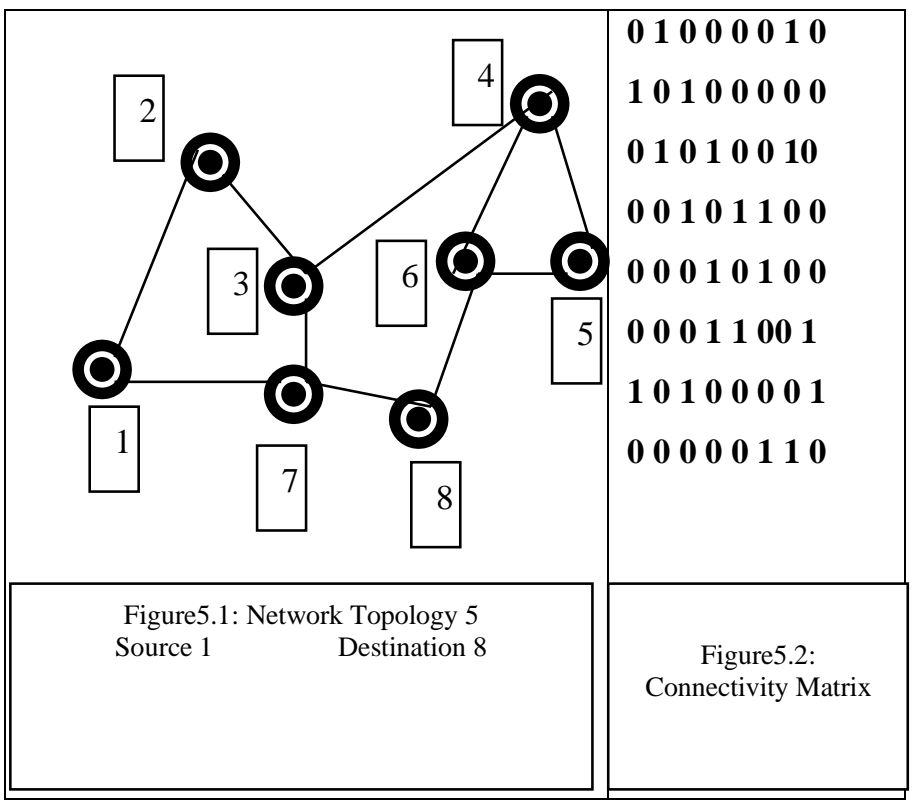

Table: 5.1: All possible path's power budget and quality factor values are given in a table.

\begin{tabular}{|cl|l|l|l|}
\hline All Possible Paths & $\begin{array}{l}\text { Length of } \\
\text { Paths }(\mathrm{km})\end{array}$ & $\begin{array}{l}\text { Power Budget } \\
(\mathrm{db})\end{array}$ & $\begin{array}{l}\text { Quality Factor } \\
(100 \%)\end{array}$ \\
\hline 1. & $1-7-8$ & 60 & 23.5 & -30 \\
\hline 2. & $1-2-3-7-8$ & 69 & 18.7 & -3.9 \\
\hline 3. & $1-2-3-4-6-8$ & 70 & 17.7 & 1.7 \\
\hline 4. & $\begin{array}{l}1-2-3-4-5- \\
6-8\end{array}$ & 94 & 7.5 & 58 \\
\hline 5. & $1-7-3-4-6-8$ & 79 & 15.3 & 15 \\
\hline 6. & $\begin{array}{l}1-7-3-4-5- \\
6-8\end{array}$ & 103 & 3.9 & 78 \\
\hline
\end{tabular}

III.

\section{SIMULATION RESULT}

We have considered network topology as shown in Figure 1.1 having 5 nodes. There our source is 1 and destination is 4.The links are shown by the line joining between two nodes. The network topology considered has the connectivity matrix as shown in Figure 1.2. We calculated power budget and quality factor for network topology which is shown in table 1.1. Following the same process, we calculate power budget and quality factor and draw a graph using following data in MATLAB. The simulation result is given below:

We get the following graph for those values of power budget which is given in table 1.1. The graph is given below:

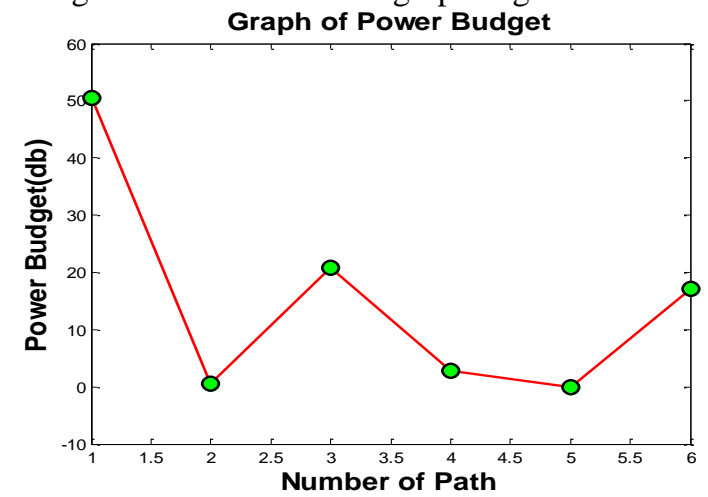

Figure 6.1: Graph of Power Budget and Reference Path Number 


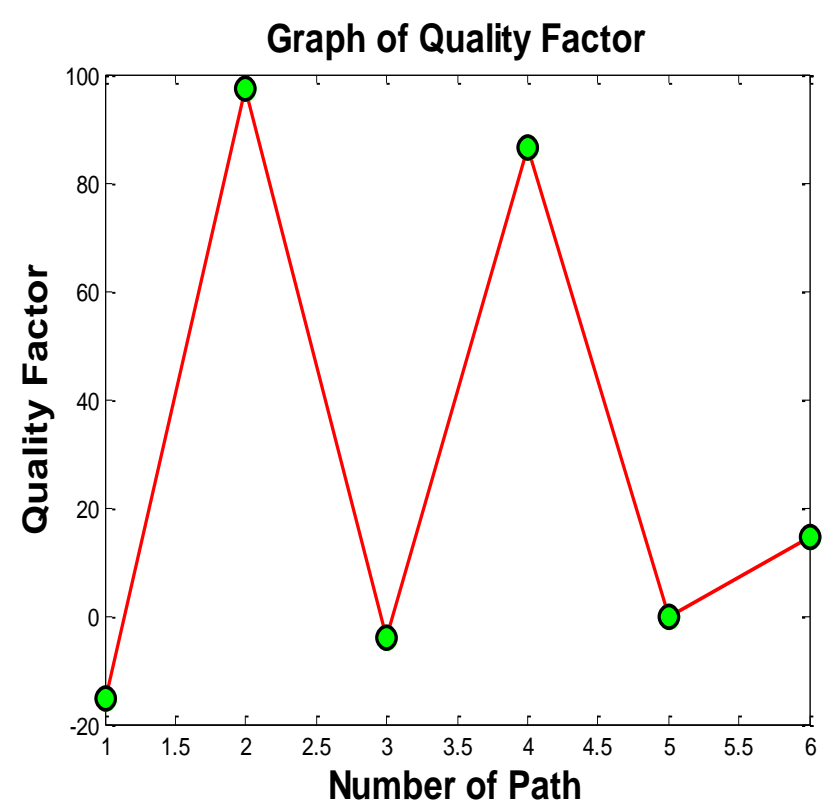

Figure 6.2: Graph of Quality Factor and Reference Path Number

We have also considered a network topology as shown in Figure 2.1 having 5 nodes. Here our source is 1 and destination is 5 . The links are shown by the line joining between two nodes. The network topology considered has the connectivity matrix as shown in Figure 2.2. We calculated power budget and quality factor for network topology which is shown in table 2.1. Following the same process, we calculate power budget and quality factor and draw a graph using following data in MATLAB. The simulation result is given below:

We get the following graph for those values of power budget which is given in table 2.1. The graph is given below:

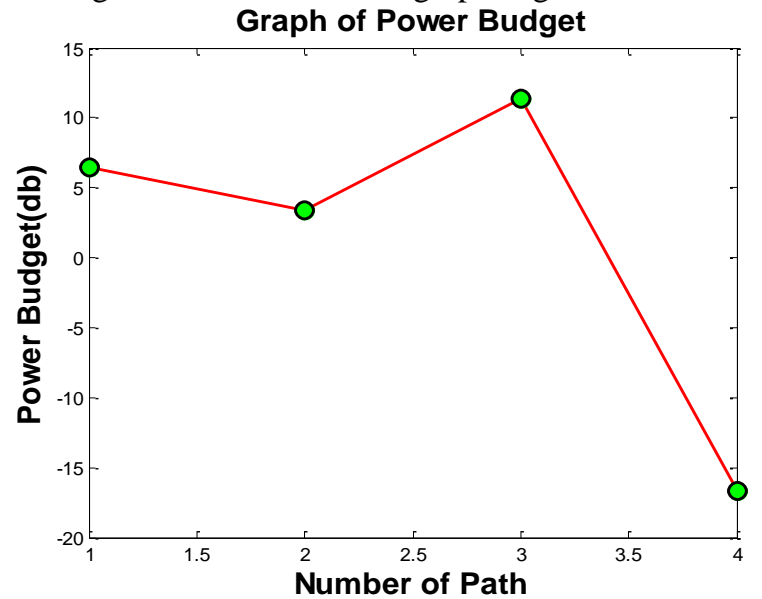

Figure 7.1: Graph of Power Budget and Reference Path Number

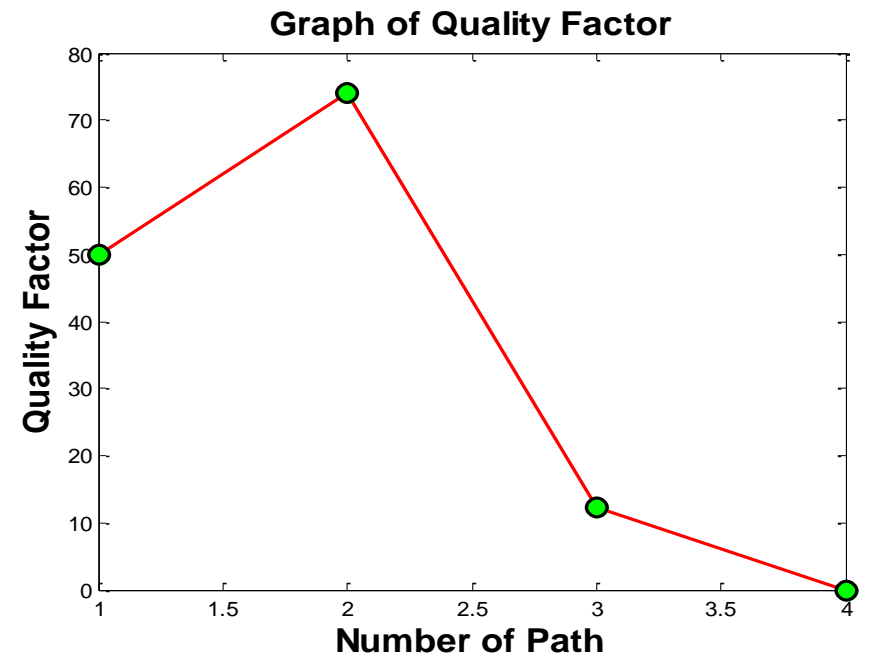

Figure 7.2: Graph of Quality Factor and Reference Path Number

We have considered network topology as shown in Figure 3.1 having 6 nodes. There our source is 1 and destination is 6.The links are shown by the line joining between two nodes. The network topology considered has the connectivity matrix as shown in Figure 3.2. We calculated power budget and quality factor for network topology which is shown in table 3.1. Following the same process, we calculate power budget and quality factor and draw a graph using following data in MATLAB. The simulation result is given below:

We get the following graph for those values of power budget which is given in table 3.1. The graph is given below:

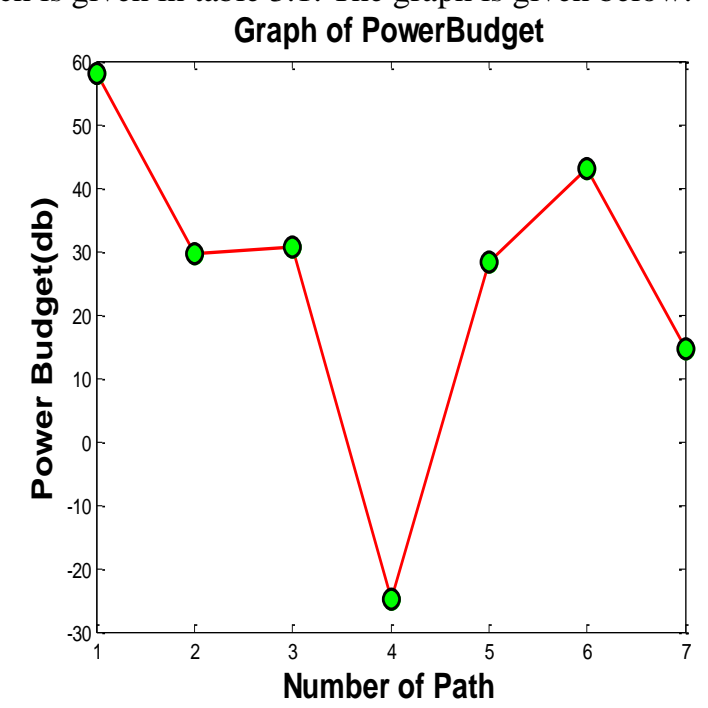

Figure 8.2: Graph of Power Budget and Reference Path Number 


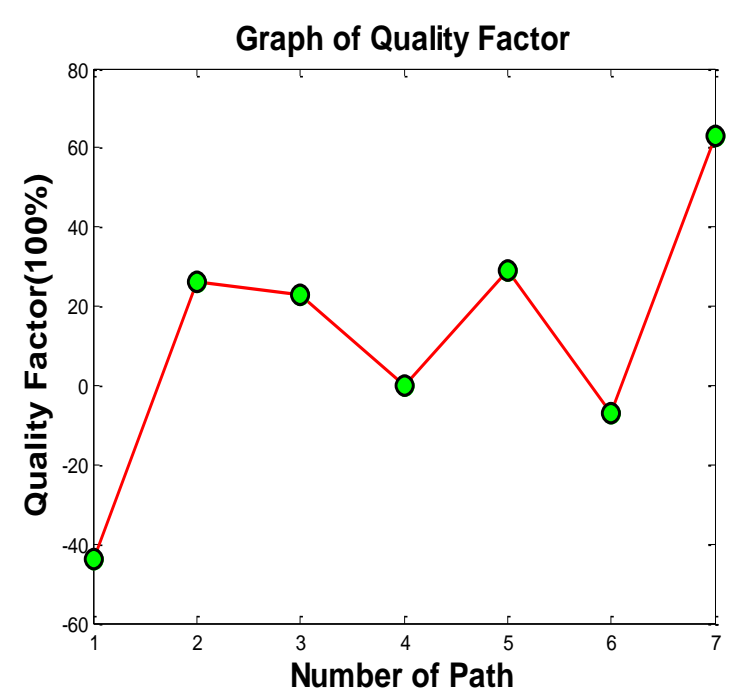

Figure 8.2: Graph of Quality Factor and Reference Path Number

We have considered network topology as shown in Figure 4.1 having 7 nodes. There our source is 1 and destination is 7.The links are shown by the line joining between two nodes. The network topology considered has the connectivity matrix as shown in Figure 4.2. We calculated power budget and quality factor for network topology which is shown in table 4.1. Following the same process, we calculate power budget and quality factor and draw a graph using following data in MATLAB. The simulation result is given below:

We get the following graph for those values of power budget which is given in table 4.1. The graph is given below:

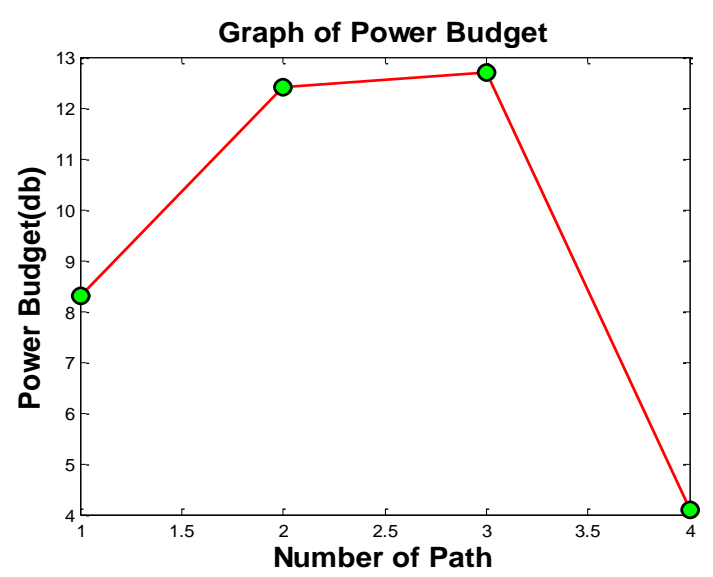

Figure 9.1: Graph of Power Budget and Reference Path Number

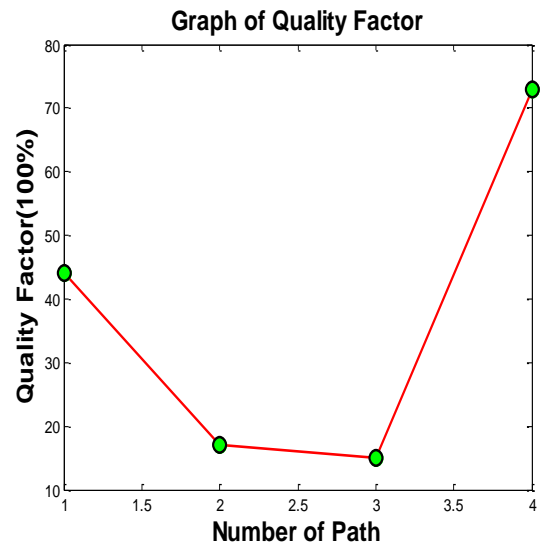

Figure 9.2: Graph of Quality Factor and Reference Path Number

We have considered network topology as shown in Figure 5.1 having 8 nodes. There our source is 1 and destination is 8.The links are shown by the line joining between two nodes. The network topology considered has the connectivity matrix as shown in Figure 5.2. We calculated power budget and quality factor for network topology which is shown in table 5.1. Following the same process, we calculate power budget and quality factor and draw a graph using following data in MATLAB. The simulation result is given below:

We get the following graph for those values of power budget which is given in table 5.1. The graph is given below:

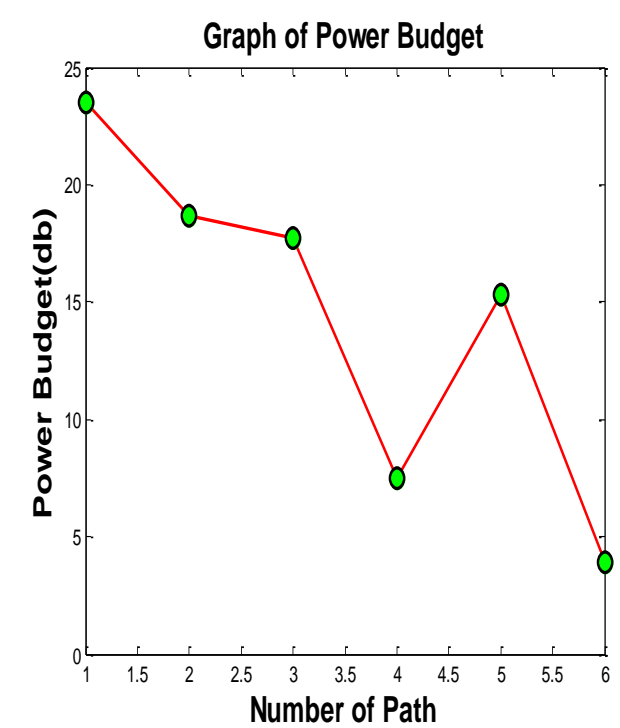

Figure 10.1: Graph of Power Budget and Reference Path Number 


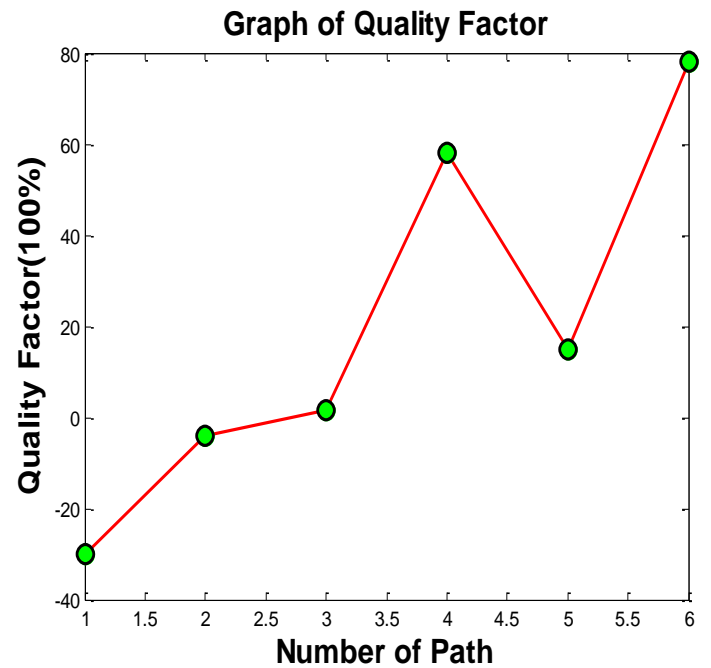

Figure 10.2: Graph of Quality Factor and Reference Path Number

Now we shall show the graph of power budget and quality factor and analyze that how they are related. The graph is given below:

We have got the first graph from the value of table 1.1.

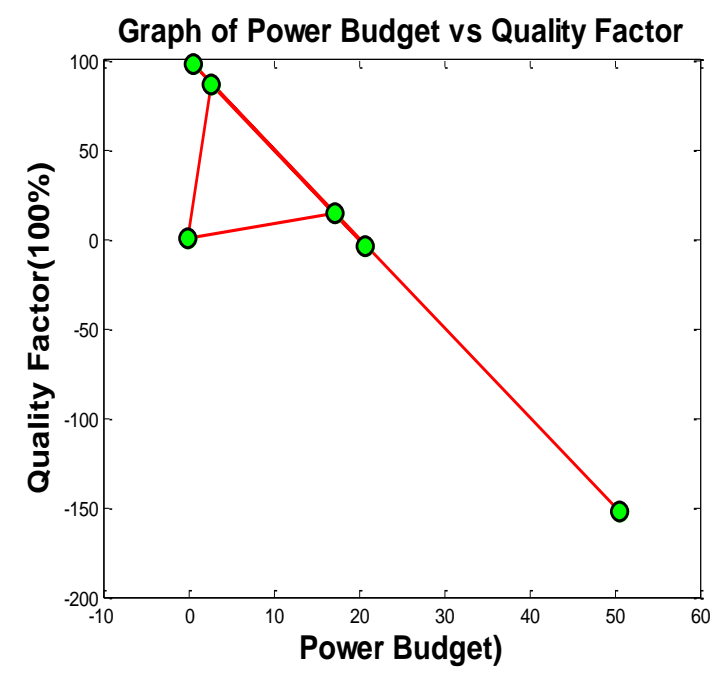

Figure 11.1: Graph of Power Budget and Quality Factor

We have got the second graph from the value of table 2.1.

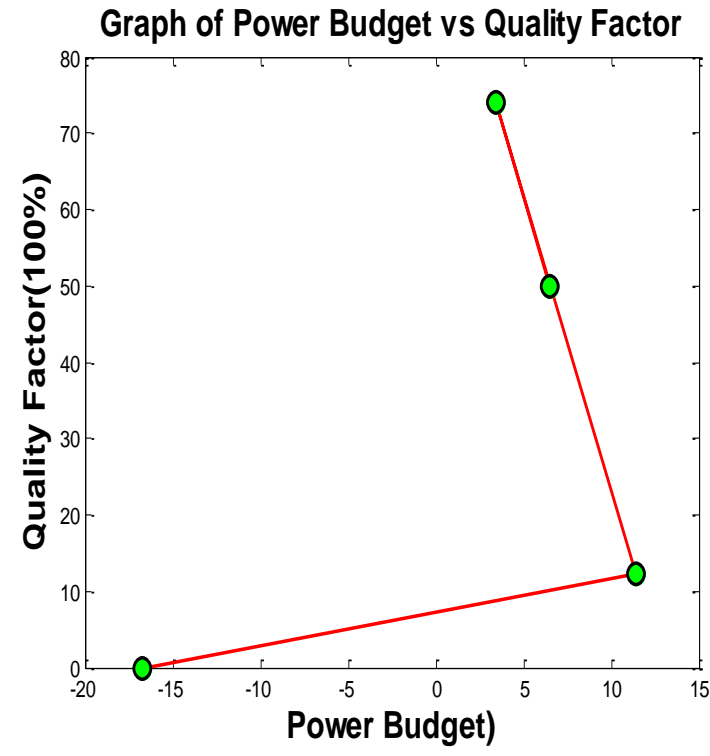

Figure 11.2: Graph of Power Budget and Quality Factor

We have got the third graph from the value of table 3.1.

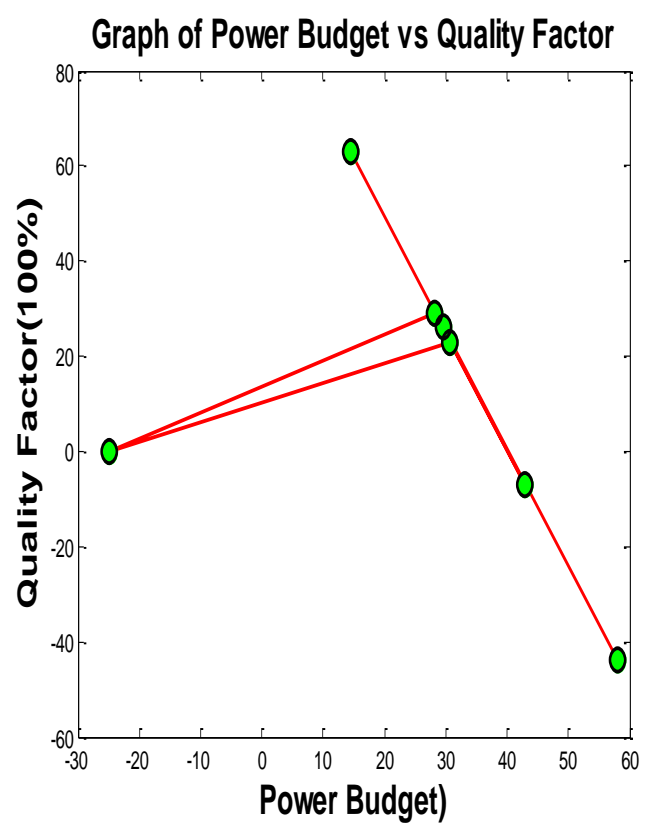

Figure 11.3: Graph of Power Budget and Quality Factor

We have got the fourth graph from the value of table 4.1 


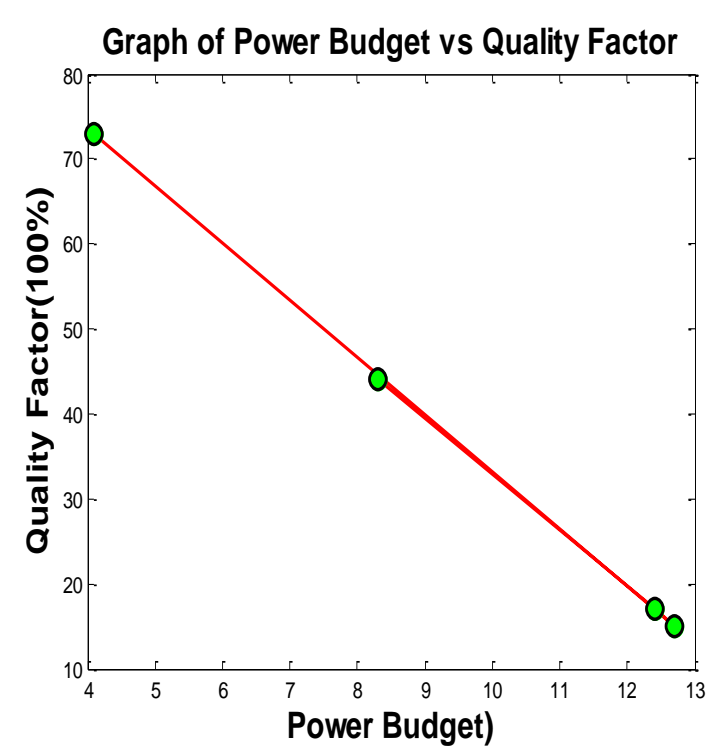

Figure 11.4: Graph of Power Budget and Quality Factor

We have got the fifth graph from the value of table 5.1.

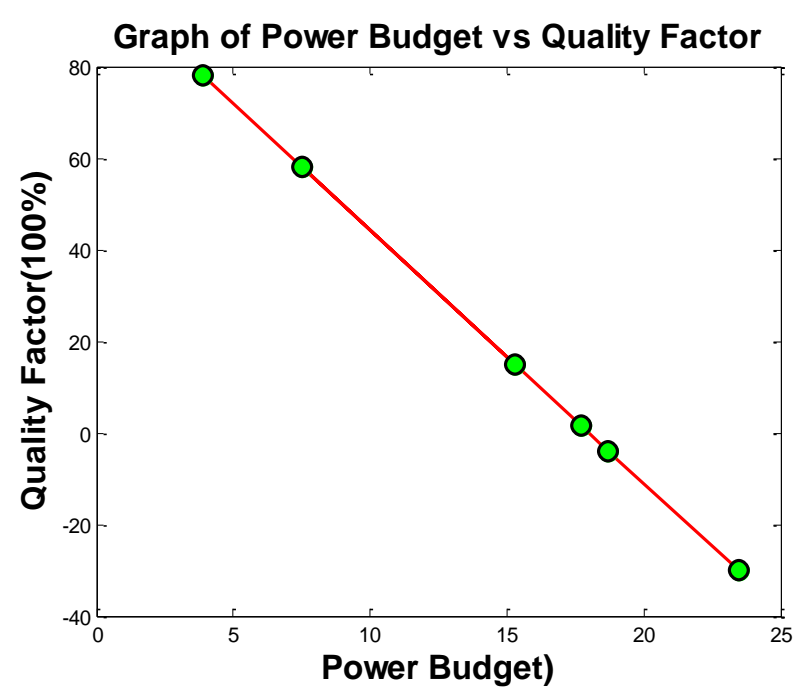

Figure 11.5: Graph of Power Budget and Quality Factor

\section{ACKNOWLEDGMENT}

I would like to express my deepest gratitude to my thesis supervisor Dr. Md. Omar Faruk for his guidance showing me the path of conducting successful research and above all for always being there as my mentor. He shared his wisdom with me in analyzing subject matters and at the same time valued my thinking approach to synthesize those topics. His suggestions drove me towards better ways of thinking, his reviews enriched me in solving problems, and his support gave me strength at the time of my disappointment. I shall forever cherish the memories of working with him. I deeply thank my friends and families for always believing in me even at the moment when I was losing my confidence.

\section{REFERENCES}

[1] https://www.techopedia.com/definition/8699/optical-fiber

[2] http//www.fiber-optic-transceiver-module.com/fiber-link-powerbudget-calculation.html

[3] https://www.fiberoptics4sale.com/blogs/archive-posts/95049798calculating-fiber-loss-and-distance-estimates

[4] https://ethesis.nitrkl.ac.in/2572/1/final_thesis.pdf

[5] https://transportgeography.org/?page_id=6969

[6] Optical fiber communication - Gerd Keiser (Mc.Graw-Hill International Edition)

[7] C.S.R. Murthy and M. Gurusamy, "WDM optical network -concepts, design and algorithms.” Upper Saddle River, NJ.: Prentice-Hall, 2002.

[8] B. Mukherjee, "Optical Communication Networks", McGraw-Hill, New York, 2003.

[9] G. Agrawal, "Fiber-Optic Communication Systems", New York, N.Y.: John Wiley \& Sons, pp. 172, 1997.

[10] John M. Senior," Optical Fiber Communications Principles and Practice", Second edition, Prentice Hall Publications.

[11] Michael J. Fujita, S.K. Ramesh, and Russell L. Tatro. "Fiber Optic Communication Link Design". 